\title{
Equanimity to Excess: Inhibiting the Expression of Negative Emotion is Associated with Depression Symptoms in Girls
}

\author{
Kate Keenan, \\ Department of Psychiatry MC 3077, University of Chicago, 5841 South Maryland Avenue, Chicago, \\ IL 60637, USA e-mail: kkeenan@yoda.bsd.uchicago.edu
}

\begin{abstract}
Alison Hipwell, Department of Psychiatry, Western Psychiatric Institute and Clinic, University of Pittsburgh, Pittsburgh, PA, USA

Amanda Hinze, and Department of Psychiatry, Western Psychiatric Institute and Clinic, University of Pittsburgh, Pittsburgh, PA, USA

Dara Babinski

Department of Psychiatry, Western Psychiatric Institute and Clinic, University of Pittsburgh, Pittsburgh, PA, USA
\end{abstract}

\section{Abstract}

Emotion dysregulation is often invoked as an important construct for understanding risk for psychopathology, but specificity of domains of emotion regulation in clinically relevant research is often lacking. In the present study Gross' (2001) model of emotion regulation is used to generate hypotheses regarding the relative contribution of two specific types of deficits in emotion regulation, inhibited and disinhibited expression of negative emotion, to individual differences in depressive symptoms in preadolescent girls. A sample of 2329 -year-old girls was recruited from a community based study. Depression symptoms were assessed via diagnostic interview. The mother and interviewer rated the girl's level of impairment. Questionnaires and observations were used to assess inhibited and disinhibited expression of negative emotion. Differences in inhibited expression of negative emotion typically explained more variance in depressive symptoms and impairment across informants than did disinhibited expression of negative emotion. Although disinhibited expression of negative emotion is associated with depression and impairment, inhibited expression appeared to be a necessary ingredient, suggesting that inhibited expression may be a particularly relevant deficit in emotion regulation in the development of depression in females.

\section{Keywords}

Depression; Girls; Emotion expression; Inhibition

\begin{abstract}
Identifying which girls are vulnerable to developing depression in adolescence from childhood characteristics has been challenging, but being able to do so is obviously of great importance to women's mental health. Prevention of depression during adolescence would be greatly aided by having reliable and valid markers that can be observed during the developmental period that precedes the onset of depression. A number of models of depression that focus on
\end{abstract}

(C) Springer Science + Business Media, LLC 2009

Correspondence to: Kate Keenan. 
precursors that are developmentally salient to preadolescent functioning in girls have been put forth (e.g., Cyranowski et al. 2000; Hankin and Abramson 2001; Keenan and Hipwell 2005; Nolen-Hoeksema 1994; Rose and Rudolph 2006). In general, these models posit that some female-specific patterns of processing and responding to social-emotional stimuli places girls at risk for depression. For example, Nolen-Hoeksema (1994), and later Hankin and Abramson (2001), proposed that girls' tendency to ruminate in response to negative emotional experiences places them at risk for depression. Rose and Rudolph (2006) speculated that girls are more likely than boys to experience negative emotions in response to their own and their friends' peer related stress, and that this may contribute to their vulnerability to depression. These models support testing the hypothesis that observable individual differences in domains such as the regulation of emotion are likely to partly explain the development of depression symptoms from preadolescence to adolescence (Keenan and Hipwell 2005).

In the present study, we utilize a conceptual framework developed by James Gross, who defined emotion regulation as "...all of the conscious and non-conscious strategies we use to increase, maintain, or decrease one or more components of an emotional response" (Gross 1999). Gross (2001) distinguished between two domains of emotion regulation: antecedent-and responsefocused emotion regulation. Antecedent-focused emotion regulation strategies include distraction, reappraisal, and modification of environments, all of which are efforts at the regulation of emotion that occur prior to the activation of an emotional response. Responsefocused emotion regulation includes efforts to regulate emotion after an emotional cue has been perceived as such and after an emotional response has been activated, such as modulating the emotional, behavioral, and or physiological response. An important component to Gross' conceptualization is that the "when" of emotion regulation vis-à-vis perception of an emotional cue is as important as the "how" of emotion regulation because strategies employed early in the process tend to be more effective than those employed later. Our interest is in the responsefocused modulation of emotion expression for two reasons. First, because children are still developing the meta-cognitive skills that are required for effective employment of antecedentfocused emotion regulation, they rely more typically on response-focused emotion regulation strategies (Yap et al. 2007). Second, as stated above, when emotion regulation strategies are engaged after the perception of an emotional cue they tend to be less effective. Therefore, individuals who report often engaging in response-focused emotion regulation strategies may experience negative emotion more frequently and or more intensely, increasing the risk for developing psychopathology.

Much of the research on emotion expression and child psychopathology is on disinhibited expression of negative emotion (often referred to as emotion dysregulation) such as frequent, intense and/or prolonged expressions of anger, reflecting a core deficit in modulation of the behavioral-physiological response to an emotional experience. There is evidence for an association between disinhibited expression of negative emotion and both disruptive behavior problems (for a review, see Keenan 2000) and depression symptoms in children (e.g., Shipman et al. 2003). In fact, newly developed therapies for depression in children are focused on helping children develop skills to "down-regulate" the disinhibited expression of sadness and anger (e.g., Kovacs et al. 2006).

Although not typically included in studies of child psychopathology, inhibited expression of negative emotion can also be conceptualized as emotion dysregulation. According to the model proposed by Gross (2001), emotion expression is inhibited when an individual encounters emotional stimuli, activates an emotional response at the physiologic, cognitive, and emotional level, but does not manifest overt behavioral indicators of that emotion. This is similar to a construct in the coping literature called disengagement (Connor-Smith and Flachsbart 2007), although an important difference is that in the case of inhibited emotion expression an 
emotional response has been activated, thus inhibition requires effort at suppressing an emotional response at multiple levels.

There are several possible mechanisms by which inhibited expression of negative emotion may be associated with the development of depression. The functionalist approach to emotion expression (Campos et al. 1989) argues that children express negative emotion in order to regulate their own emotions and to communicate effectively with others. As such, healthy social-emotional functioning is contingent on being able to express one's emotions in a culturally appropriate way (Cole et al. 1994). The inhibition of the expression of negative emotion may lead to an inability to effectively communicate emotional experiences to others, resulting in a lack of social support for managing negative emotions, and a lack of opportunity to develop skills to regulate negative emotions once they are expressed. Lack of inter-and intrapersonal strategies for managing one's response to emotional provocations could lead to a state of chronic dysphoria or feelings of helplessness and hopelessness in coping with negative emotions. Inhibition of negative emotion also may reflect a lack of assertiveness or belief in the importance of one's emotional response to a stimulus, both of which may be liabilities for meeting the demands of autonomy and self-identity that increase during adolescence, thereby conferring risk for maladjustment to adolescent stressors.

There are data suggesting that there is a point at which inhibited expression of emotion has implications for the development of psychopathology. Gross and John (2003) demonstrated negative concurrent associations between self-reports of "habitual" suppression of emotion and autonomy, mastery, and optimism, and a positive association with depression. In an experimental study, Richards and Gross (1999) demonstrated that compared to emotion expression, suppression of emotion was associated with less accurate performance and increased sympathetic activation of the cardiovascular system during a memory task. Data from two studies of females provide further support for the hypothesis that suppression of negative emotion has negative consequences. In an experimental manipulation of emotion expression among unacquainted pairs of young women, Butler and et al. (2003) reported that in comparison to women who expressed negative emotion women who suppressed negative emotion during the discussion of an upsetting topic were viewed as less socially engaging. Bromberger and Matthews (1996) reported that, compared with women who expressed their angry feelings, women who reported holding their anger in had higher depression scores 3 years later. Finally, in one the few relevant studies of children, Silk and et al. (2003) used an experience sampling method to test associations between emotion regulation strategies, emotion intensity, and depression symptoms in 12-and 15-year olds. Youth-reported disengagement (e.g., denial, avoidance, wishful thinking, inaction) on the Response to Stress Questionnaire (Connor-Smith et al. 2000) was associated with higher levels of anger and sadness and depression symptoms assessed by self-report on a questionnaire.

Inhibition of emotion expression may be particularly relevant for the development of depression in females (Keenan and Hipwell 2005). Although there are little to no sex differences in basic emotional processes (Gross and Levenson 1993), there do appear to be sex differences in the interpersonal communication of emotion. For example, results from several studies using emotional provocation demonstrate that girls are more likely than boys to inhibit the expression of anger, frustration, and sadness (Cole 1986; Saarni 1984; Underwood et al. 1999). This is particularly true for anger, but also for disappointment and sadness, especially when being observed by others (Saarni 1984). In a study of college students, females were more likely than males to express happiness during a frustrating task with a friend as opposed to a negative emotion, and this incongruent expression of happiness was associated with depression symptoms (Chaplin 2006). Sex differences in the suppression or masking of negative emotions appear to be due in part to differences in how boys and girls are socialized to express emotion. For example, there is evidence that girls, more often than boys, are 
socialized by parents and teachers to consider the impact of the expression of negative emotions on the emotional well being of others (Keenan and Shaw 1997). Girls are more likely than boys to anticipate negative reactions from others in response to their expression of negative emotions (e.g., Underwood 1997). Clues to precursors to depression in girls are likely to be revealed in data such as these on sex differences in the development of psychological processes.

These two sets of empirical findings, that girls are more likely than boys to inhibit the expression of negative emotion and that this strategy for regulating emotion can have negative consequences for psychological functioning, form the rationale upon which the present study is based. We tested whether disinhibited and inhibited expressions of sadness and anger were differentially associated with emerging depression symptoms and impairment in preadolescent girls using two independent measures of emotion expression: self-report and observations. Evidence for the utility of inhibition of emotion expression in a developmentally informed model of depression in females would be supported by a positive association with individual differences in depressive symptoms and impaired functioning prior to adolescence.

Furthermore, we hypothesized that inhibited expression of negative emotion would explain more variance in depression symptoms and impairment than would disinhibited expression of negative emotion.

\section{Method}

\section{Sampling of Participants}

Participants were 232 girls and their biological mothers who were recruited from a longitudinal study of girls who are representative of those living in the city of Pittsburgh. Participants were identified via a household enumeration in which all homes in low income neighborhood and $50 \%$ of home in middle to upper-income neighborhoods were enumerated. Girls selected for participation in the present study were from the youngest participants in the community sample who either screened high on measures of depression symptoms by self-report on the Short Moods and Feelings Questionnaire (Angold et al. 1995) and maternal-report on the Child Symptom Inventory (Gadow and Sprafkin 1996) at age eight, or who were included in a random selection from the remaining girls. This sampling strategy was used in order to increase the base rate of depression as the girls moved into adolescence. Girls whose scores fell at or above the 75th percentile by their own report, the mother's report or by both informants comprised the screen high group $(n=135)$. There were significantly more African American than European American girls in the screen high group. Thus, the girls selected from the remainder were matched to the screen high group on race $(n=136)$. Eight families were not eligible at the time of recruitment because the biological mother had died, the family had moved out of state, or the family was no longer participating in the community study and could not be contacted. Letters were mailed and subsequent phone calls were made to the 263 remaining eligible families, of whom $232(88.2 \%)$ agreed to participate and completed the laboratory assessment, $25(9.5 \%)$ refused to participate, and $6(2.3 \%)$ agreed but could not be scheduled for an assessment with the required timeframe.

When the girls were 9 years of age, they and their mothers completed a laboratory assessment during which each informant independently provided information on DSM-IV symptoms of depression, completed questionnaires, and were observed during structured tasks. Written informed parental consent and child assent were obtained. The University of Pittsburgh Institutional Review Board approved all study procedures.

\section{Measures}

Current symptoms of depression were measured using the Schedule for Affective Disorders and Schizophrenia for School-Age Children-Present and Lifetime Version (K-SADS-PL; 
Kaufman et al. 1997), a semi-structured diagnostic interview administered to both the girl and her mother. All nine symptoms of depression were asked regardless of endorsement of disturbance in mood (i.e. dysphoria or irritability) or anhedonia. We used a count of depression symptoms endorsed at the level of threshold on the K-SADS as the dependent measure of depression symptoms. A second interviewer listened to, and coded, responses from the digital video of the K-SADS-PL interview to assess inter-rater agreement. Twenty-five percent of the interviews with the mothers and with the girls were randomly selected for this purpose. The intraclass correlations coefficient (ICC) was 0.91 for maternal-and 0.92 for youth-reported symptoms.

Emotion expression was assessed via child report using the Children's Sadness/Anger Management Scale (CSMS/CAMS; Zeman et al. 2001) and the Emotion Expressive Scale (EESC; Penza-Clyve and Zeman 2002). The CSMS/CAMS are youth-report measures designed for elementary school-age children. Each item is rated on a three-point scale. For the purposes of the present study, scores on the dysregulated factor (e.g., I do things like slam doors when I'm mad; I do things like mope around when I'm sad) were used to measure disinhibited expression of negative emotion (sample mean=11.06, $\mathrm{SD}=2.2$ ). The alpha for the six-item measure of disinhibition was 0.47 . Although this level of internal consistency was relatively low, it is consistent with reported alphas for other brief child-report measures (e.g., Sodano and Tracey 2006), and is comparable to that reported for the emotion regulation subscale (alpha $=0.48)$ on the Response to Stress Questionnaire (Connor-Smith et al. 2000) in a similar socio-demographic sample.

The EESC is a 16-item self-report measure yielding scores on two factors: poor awareness and expressive reluctance. Each item is rated on a five-point scale. The scale was validated using 9-12 year old children and has demonstrated internal consistency, test-retest reliability, and construct validity (Penza-Clyve and Zeman 2002). In the present study, scores on the 8-item expressive reluctance factor (e.g., when I'm sad I try not to show it; when I get upset I'm afraid to show it) were used as a measure of inhibited expression of negative emotion (sample mean=17.72, SD=6.4). The alpha for this scale was 0.80 . Scores on self-reported inhibited and disinhibited emotion expression were positively correlated (Pearson correlation coefficient $=0.33, p<0.001$ ).

Observed emotion expression was coded during a family problem-solving task, during which the girl and her mother each generated a topic of conflict and then discussed each topic for 3 min. Digital recordings were coded by a team of raters who were unaware of the girls' responses on any questionnaire or interview. Nine-point scales developed by Melnick and Hinshaw (2000) were used to generate global ratings based on the entire problem-solving task of disinhibited and inhibited expressions of negative emotion. Vocalizations, facial displays, and posture were used to generate codes of disinhibition and inhibition of anger, sadness, and frustration, with higher scores indicating higher levels of the observed emotion expression. Inhibited expression included behaviors indicating disengagement, distancing, and/or completely shutting down. Disinhibited emotion expression included behaviors indicative of anger, frustration, disappointment, and/or sadness. Ratings on these two scales were positively correlated (Pearson correlation coefficient $=0.18, p<0.01$ ). Observed and self-reported inhibited and disinhibited expressions of emotion were not significantly correlated.

A random selection of $25 \%$ of the digital recordings of the problem solving task were coded by a second rater to assess inter-rater reliability: the ICC was 0.89 and 0.87 for disinhibited and inhibited expression of negative emotion, respectively.

The Children's-Global Assessment Scale (C-GAS; Setterberg et al. 1992) is a measure of impairment developed for children 4-18 years of age, which was completed by the mother and 
the interviewer following the administration of the K-SADS. Scores on the C-GAS range from 1 to 100 with each decile containing a description of the severity of symptomatology in terms of the impact the symptoms have on school, family, and peer relations. Lower scores on this scale reflect greater impairment. Inter-rater agreement on 42 cases of interviewer-generated C-GAS ratings following the child K-SADS was very high: intraclass correlation coefficient (ICC=0.91).

\section{Results \\ Depression Symptoms}

Very few 9-year-old girls met criteria for major depression by either youth $(n=16,6.9 \%)$ or maternal report $(n=8,3.4 \%)$. Although these rates are higher than one would expect to find in an epidemiologic sample, demonstrating the success of the sampling strategy, they were not sufficiently high for use as a dependent measure of depression. Sufficient variability was observed, however, in the distribution of depression symptoms. By youth report, having one or more symptoms of depression was more common than having no symptoms. Eighty-four girls $(36.2 \%)$ reported no symptoms of depression, 51 (22.0\%) reported one symptom, 39 $(16.8 \%)$ reported two symptoms, $38(16.4 \%)$ reported three to four symptoms, and 20 girls (8.6\%) reported five or more symptoms. Maternal report of girls' symptoms produced lower rates: 99 (42.7\%) girls had no symptoms of depression, 54 (23.3\%) had one symptom, 40 (17.2\%) had two symptoms, $33(14.2 \%)$ had 3-4 symptoms, and six (2.6\%) had five or more symptoms. Because the magnitude of association between youth and maternal report was relatively low ( $\mathrm{ICC}=0.26$ ) and because there is an advantage to testing associations between emotion expression and depression symptoms within and across informants, youth and maternal reports of depression symptoms were analyzed separately.

\section{Univariate Tests of Association Between Emotion Expression and Depression Symptoms and Impairment}

Youthreported emotion expression was significantly associated with youth report and maternal report of depression symptoms, and maternal and interviewer C-GAS ratings in the expected directions ranging in magnitude from -0.13 for maternal-reported impairment and youth report of disinhibited emotion expression to 0.52 for youth-reported MDD symptoms and youthreported inhibited emotion expression (see Table 1), with positive correlations indicating that higher levels of inhibition are associated with higher levels of depression symptoms and negative correlations indicating that higher levels of inhibition are associated with lower levels of functioning. Fewer significant associations were found between observed emotion expression and MDD symptoms and impairment. Maternal-reported impairment was significantly associated with observed disinhibited emotion expression (Spearman's rho= $-0.12, p<0.05)$. Observed inhibited emotion expression was significantly, although modestly, associated with maternal-reported MDD symptoms (Spearman's $r h o=0.15, p<0.05$ ) and interviewer-and maternal-reported impairment (Spearman's $r h o=-0.13, p<0.05$ and -0.16 , $p<0.01$, respectively) (Table 1 ).

\section{Multivariate Tests of Associations Between Emotion Expression and Depression Symptoms and Impairment}

To determine whether youth-reported and observed inhibited and disinhibited emotion expression accounted for unique variance in depression and impairment, linear regressions were computed using a forward, stepwise selection method; statistics for variables not included in the final model also are included in each table. Results for MDD symptoms are presented in Table 2. Approximately $24 \%$ of the variance in youth reported depression symptoms was accounted for by youth-reported inhibited expression of negative emotion, and an additional $4 \%$ was accounted for by youth-reported disinhibited expression $(F[2,227]=44.57, p<0.001$, 
adjusted $R$ square $=0.276$ ). Youth-reported inhibited expression of negative emotion explained a small amount of variance in maternal report of MDD symptoms $(F[1,228]=12.57, p<0.01$, adjusted $R$ square $=0.048$ ), whereas measures of disinhibited emotion expression did not significantly add to this model.

As shown in Table 3, close to $20 \%$ of the variance in interviewer rated impairment was accounted for by youth reported inhibited emotion expression, with an additional $5 \%$ attributed to disinhibited expression $(F[2,227]=38.41, p<0.001$, adjusted $R$ square $=0.256)$. Individual differences in maternal-rated impairment were modestly associated with observed and youthreported inhibited emotion expression $(F[2,227]=12.57, p<0.01$, adjusted $R$ square $=0.040)$. Of the four models, this was the only one in which an observed measure of emotion expression explained unique variance (Table 3).

\section{Discussion}

In an effort to develop a set of preadolescent precursors to adolescent onset depression in girls, we examined the relative association of inhibited and disinhibited expression of negative emotion to depression symptoms and impairment in 9-year old girls. Very few girls met criteria for DSM-IV MDD in the present sample, but sufficient variability in the number of depression symptoms endorsed was observed in both youth and maternal report. Thus, we are capturing a developmental period in which individual differences in the level of depression symptoms can be measured, as well as a period that precedes the major risk period for MDD.

Both inhibited and disinhibited patterns of emotion expression were associated with depression symptoms and impairment. This was first revealed in the univariate analyses, and reinforced to some extent by the multivariate analyses. Of the variance explained by emotion expression, however, youth-reported inhibition of the expression of negative emotion accounted for the majority. Overall, inhibition of negative emotion appeared to be a necessary ingredient for explaining variance in youth-, maternal-, and interviewer-reported outcomes. Thus, our stated criteria of the utility of inhibition of emotion expression in a developmentally informed model of depression in females, namely a positive association with individual differences in depression symptoms and impaired functioning prior to adolescence, was met. This finding is important because the majority of research on emotion regulation and psychopathology in children has been focused on disinhibited negative emotion. The results from the present study suggest that inhibition of anger and sadness may be an equally salient, if not more salient, deficit in emotion regulation for the development of depression symptoms and impairment among girls.

A number of measurement issues, however, temper these results. First, the reliability of the self-report measure of disinhibition was lower than the measure of inhibition and was less than optimal. This difference in reliability may have affected the results for the self-report measures, but would not have affected the results from the observed measures given the equality in reliability for those measures. Moreover, at the univariate level, the magnitude of the association between self-reported disinhibited emotion expression and depression and impairment, was comparable to that between inhibited emotion expression and depression and impairment. Thus, internal consistency of the measure is unlikely to have impacted on the multivariate analyses. Second, the design of the present study did not allow us to test whether results can be generalized across negative and positive emotions or if they are specific to a single negative emotion (e.g., anger versus sadness). The third measurement issue was that self - report and observed measures of emotion expression were not significantly associated. This is not surprising given that the magnitude of association between observed and rated traits and symptoms in children is often low (e.g., Klinnert et al. 2000). In addition, self-rated and observed measures of emotion expression were confounded with context: girls reported on 
their typical style of emotion expression and then were observed in a specific stressful context of discussing a disagreement with their mothers. Garber et al. (1995) found that children's reports of emotion regulation strategies vary depending on the context (e.g., fight with a friend versus doing poorly at a game), and that the interaction of sex and depression symptoms on emotion regulation strategies also varied by context. A lack of significant association, therefore, should not lead to the conclusion that either measure lacked validity. However, the results do highlight the importance of developing equivalent sampling of behaviors across informants or methods of assessment, such as assessing emotion expression in the context of the mother-child relationship separately from relationships with peers.

Interestingly, within each method of assessment emotion inhibition and disinhibition was positively correlated. This suggests that some girls manifest multiple deficits in the regulation of emotion via emotion expression, and both types of deficits were associated with symptoms of depression. Exploring whether there is a temporal association between these deficits will be important for determining their respective roles in conferring risk for depression. For example, some girls may engage in inhibition of negative emotion as a result of negative consequences following disinhibited emotion expression. A significant association between inhibited and disinhibited emotion expression may also result from girls whose tendencies to inhibit negative emotion are difficult to maintain, and as a result they engage in infrequent, but intense displays of disinhibited expression.

Because maternal and youth reports of depression symptoms and MDD were not highly correlated, we chose to test the association with deficits in emotion expression separately by informant. It is perhaps not surprising that the pattern of association was more robust when the same informant was used for the independent and dependent measures. On the other hand, the fact that youth-report and observed inhibited expression of emotion also was associated with mother reported depression symptoms and impairment, albeit modestly, provides refutation against the argument that the findings are the result of common methods bias.

The results of the present study are limited to testing concurrent associations with depression symptoms. Although this is an important first step, establishing a prospective association between inhibition of negative emotions in the preadolescent period and depressive disorders in adolescence is the ultimate goal. If such support is generated, then elucidating earlier childhood pathways to this type of deficit in emotion expression/regulation will be indicated. Eisenberg et al. (1996) have demonstrated that children of parents who respond to children's negative emotions by threatening punishment or by being dismissive are more likely to engage in avoidant coping strategies in response to a problem. Results such as these in combination with the literature on sex differences in emotion socialization (e.g., Chaplin et al. 2005; Morris et al. 2007) suggest that girls who are higher than average in the expression of negative emotion, and who are socialized via punishment or dismissal to inhibit the expression of their negative emotions may be at highest risk for adolescent onset depression. Longitudinal designs that begin early in childhood and measure ease of elicitation and intensity of negative emotion, as well as parental response to emotion expression and emerging emotion regulation skills are needed to test this proposed developmental pathway to depression.

Finally, it is important to consider whether there may be contexts in which inhibition of negative emotion is adaptive. For example, there is new evidence in the adult literature that coping with a trauma, such as the death of a spouse, by repressing negative emotions is associated with better psychological and health outcomes than coping via expression of negative emotion (Coifman et al. 2007). King and Emmons $(1990,1991)$ have proposed and provided some evidence from studies with adults for the hypothesis that congruency between emotion style and emotion expression, as opposed to emotion expression in and of itself, determines psychological and physical well being. Lack of emotion expression, according to their 
hypothesis, would not confer risk for depression among those who tend to be alexithymic or low on emotionality. This is highly consistent with Gross' (2001) model, which posits that adult health is affected when negative emotion is experienced but not expressed. It may follow that the match between children's typical emotional style and their level of emotion expression is the critical variable to measure. These two variables are difficult to disentangle, however, because they are often confounded when they are measured. Moreover, longitudinal data beginning early in life would be required to capture a child's temperamental disposition toward emotion expression before manifestations of that disposition are socialized.

Another approach to addressing the link between emotion regulation and depression is by incorporating coping styles into the model. Compas et al. (2004) characterized two sets of coping styles or processes: automatic and controlled, and argued that temperament, including emotionality, can either facilitate or constrain controlled efforts to cope with stress. When coping is impeded under conditions of acute or chronic stress, depression is a likely outcome (Compas et al. 2004).

As is true for many indices of stress response and reactivity, the key to long-term mental health is likely to be determined in part by flexible systems that allow the individual to optimally respond to a stimulus given contextual demands and individual resources. Thus, our hypothesis that a pattern of inhibition of negative emotion during preadolescence confers risk for depression will ultimately require the assessment of chronic and acute stressors over time, and whether patterns of emotion expression co-vary with changes in environmental stressors or demands.

\section{Acknowledgments}

This study was funded by grant R01 MH66167 from the National Institute of Mental Health to Dr Keenan. The authors thank the families participating in the Learning About Girls' Emotions Study.

\section{References}

Angold A, Costello EJ, Messer SC, Pickles A. Development of a short questionnaire for use in epidemiological studies of depression in children and adolescents. International Journal of Methods in Psychiatric Research 1995;5:237-249.

Bromberger JT, Matthews KA. A "feminine" model of vulnerability to depression symptoms: A longitudinal investigation of middle-aged women. Journal of Personality and Social Psychology 1996;70:591-598. [PubMed: 8851743]

Butler EA, Egloff B, Wilhelm FH, Smith NC, Erickson EA, Gross JJ. The social consequences of expressive suppression. Emotion (Washington, D.C.) 2003;3:48-67.

Campos JJ, Campos RG, Barrett KC. Emergent themes in the study of emotional development and emotion regulation. Developmental Psychology 1989;25:394-402.

Chaplin TM. Anger, happiness, and sadness: Associations with depression symptoms in late adolescence. Journal of Youth and Adolescence 2006;35:977-986.

Chaplin TM, Cole PM, Zahn-Waxler C. Parental socialization of emotion expression: Gender differences and relations to child adjustment. Emotion (Washington, D.C.) 2005;5:80-88.

Coifman KG, Bonanno GA, Ray RD, Gross JJ. Does repressive coping promote resilience? Affectiveautonomic response discrepancy during bereavement. Journal of Personality and Social Psychology 2007;92:745-758. [PubMed: 17469956]

Cole PM. Children's spontaneous control of facial expression. Child Development 1986;57:1309-1321.

Cole PM, Michel MK, Teti LO. The development of emotion regulation and dysregulation: A clinical perspective. Monographs of the Society for Research in Child Development 1994;59:73-100. [PubMed: 7984169] 
Compas BE, Connor-Smith J, Jaser SS. Temperament, stress reactivity, and coping: Implications for depression in childhood and adolescence. Journal of Clinical Child and Adolescent Psychology 2004;33:21-31. [PubMed: 15028538]

Connor-Smith JK, Flachsbart C. Relations between personality and coping: A meta-analysis. Journal of Personality and Social Psychology 2007;93:1080-1107. [PubMed: 18072856]

Connor-Smith JK, Compas BE, Wadsworth ME, Thomsen AH, Saltzman H. Responses to stress in adolescence: Measurement of coping and involuntary stress responses. Journal of Consulting and Clinical Psychology 2000;68:976-992. [PubMed: 11142550]

Cyranowski JM, Frank E, Young E, Shear K. Adolescent onset of the gender difference in lifetime rates of major depression. Archives of General Psychiatry 2000;57:21-27. [PubMed: 10632229]

Eisenberg N, Fabes RA, Murphy BC. Parents' reactions to children's negative emotions: Relations to children's social competence and comforting behavior. Child Development 1996;67:2227-2247. [PubMed: 9022240]

Gadow, KD.; Sprafkin, J. Child symptom inventory. Stony Brook, NY: State University of New York at Stony Brook; 1996.

Garber J, Braafladt N, Weiss B. Affect regulation in depressed and nondepressed children and young adolescents. Development and Psychopathology 1995;7:93-115.

Gross, JJ. Emotion and emotion regulation. In: Pervin, LA.; John, OP., editors. Handbook of personality: Theory and research. Vol. 2nd ed.. New York, NY: Guilford: 1999. p. 525-552.

Gross JJ. Emotion regulation in adulthood: Timing is everything. Current Directions in Psychological Science 2001;10:214-219.

Gross JJ, Levenson RW. Emotional suppression: physiology, self-report, and expressive behavior. Journal of Personality and Social Psychology 1993;64:970-986. [PubMed: 8326473]

Gross JJ, John OP. Individual differences in two emotion regulation processes: Implications for affect, relationships, and well-being. Journal of Personality and Social Psychology 2003;85:348-362. [PubMed: 12916575]

Hankin BL, Abramson LY. Development of gender differences in depression: An elaborated cognitive vulnerability-transactional stress theory. Psychological Bulletin 2001;127:773-796. [PubMed: $11726071]$

Kaufman J, Birmaher B, Brent DA, Rao U, Flynn C, Moreci P, et al. Schedule for affective disorders and schizophrenia for school-age children-present and lifetime version (K-SADS-PL): Initial reliability and validity data. Journal of the American Academy of Child and Adolescent Psychiatry 1997;36:980-988. [PubMed: 9204677]

Keenan K. Emotion dysregulation as a risk factor for child psychopathology. Clinical Psychology: Science and Practice 2000;7:418-434.

Keenan K, Shaw DS. Developmental and social influences on young girls' behavioral and emotional problems. Psychological Bulletin 1997;121:97-113.

Keenan K, Hipwell AE. Preadolescent clues to understanding depression in girls. Clinical Child and Family Psychology Review 2005;8:89-105. [PubMed: 15984082]

King LA, Emmons RA. Conflict over emotional expression: Psychological and physical correlates. Journal of Personality and Social Psychology 1990;58:864-877. [PubMed: 2348373]

King LA, Emmons RA. Psychological, physical, and interpersonal correlates of emotional expressiveness, conflict, and control. European Journal of Personality 1991;5:131-150.

Klinnert MD, McQuaid EL, McCormick D, Adinoff AD, Bryant NE. A multi-method assessment of behavioral and emotional adjustment in children with asthma. Journal of Pediatric Psychology 2000;25:35-46. [PubMed: 10826242]

Kovacs M, Sherrill J, George CJ, Pollock M, Tumuluru RV, Ho V. Contextual Emotion-Regulation Therapy for childhood depression: Description and pilot testing of a new intervention. Journal of the American Academy of Child and Adolescent Psychiatry 2006;45:892-903. [PubMed: 16865031]

Melnick SM, Hinshaw SP. Emotion regulation and parenting in AD/HD and comparison boys: Linkages with social behaviors and peer preference. Journal of Abnormal Child Psychology 2000;28:73-86. [PubMed: 10772351]

Morris AS, Silk JS, Steinberg L, Myers SS, Robinson LR. The role of the family context in the development of emotion regulation. Social Development 2007;16:361-388. 
Nolen-Hoeksema S. An interactive model for the emergence of gender. Journal of Research on Adolescence 1994;4:519-534.

Penza-Clyve S, Zeman J. Initial validation of the Emotion Expression Scale for children (EESC). Journal of Clinical Child and Adolescent Psychology 2002;31:540-547. [PubMed: 12402572]

Richards JM, Gross JJ. Composure at any cost? The cognitive consequences of emotion suppression. Personality and Social Psychology Bulletin 1999;25:1033-1044.

Rose AJ, Rudolph KD. A review of sex differences in peer relationship processes: Potential trade-offs for the emotional and behavioral development of girls and boys. Psychological Bulletin 2006;132:98131. [PubMed: 16435959]

Saarni C. An observational study of children's attempts to monitor their expressive behavior. Child Development 1984;55:1504-1513.

Setterberg, S.; Bird, H.; Gould, M.; Shaffer, D.; Fisher, P. Parent and interviewer versions of the Children's Global Assessment Scale. New York: Columbia University; 1992.

Shipman KL, Zeman J, Nesin AE, Fitzgerald M. Children's strategies for displaying anger and sadness: What works with whom? Merrill-Palmer Quarterly 2003;49:100-122.

Silk JS, Steinberg L, Morris AS. Adolescents' emotion regulation in daily life: Links to depression symptoms and problem behavior. Child Development 2003;74:1869-1880. [PubMed: 14669901]

Sodano SM, Tracey TJG. Interpersonal traits in childhood: Development of the Child and Adolescent Interpersonal Survey. Journal of Personality Assessment 2006;87:317-329. [PubMed: 17134339]

Underwood MK. Peer social status and children's understanding of the expression and control of positive and negative emotions. Merrill-Palmer Quarterly 1997;43:610-634.

Underwood MK, Hurley JC, Johanson CA, Mosley JE. An experimental, observational investigation of children's responses to peer provocation: Developmental and gender differences in middle childhood. Child Development 1999;70:1428-1446. [PubMed: 10621965]

Yap MBH, Allen NB, Sheeber L. Using an emotion regulation framework to understand the role of temperament and family processes in risk for adolescent depression disorders. Clinical Child and Family Psychology Review 2007;10:180-196. [PubMed: 17265137]

Zeman J, Shipman K, Penza-Clyve S. Development and initial validation of The Children's Sadness Management Scale. Journal of Nonverbal Behavior 2001;25:187-205. 
Table 1

Univariate Tests of the Association Between Emotion Expression and MDD Symptoms and Impairment

\begin{tabular}{|c|c|c|c|c|}
\hline & \multicolumn{2}{|l|}{ Disinhibited } & \multicolumn{2}{|l|}{ Inhibited } \\
\hline & Youth-report & Observed & Youth-report & Observed \\
\hline MDD symptoms (youth report) & $0.41^{* * * *}$ & -0.02 & $0.52^{* * *}$ & 0.03 \\
\hline MDD symptoms (parent report) & $0.25^{* * *}$ & 0.07 & $0.24^{* * *}$ & $0.15^{*}$ \\
\hline Impairment (interviewer report) & $-0.41^{* * *}$ & -0.02 & $-0.47^{* * *}$ & $-0.13^{*}$ \\
\hline Impairment (parent report) & $-0.13^{*}$ & $-0.12^{*}$ & $-0.17^{* *}$ & $-0.16^{* *}$ \\
\hline
\end{tabular}

Spearman's rho for non-parametric data:

* $p<0.05$;

** $p<0.01$;

*** $p<0.001$ 
Table 2

Multivariate Tests of the Associations Between Emotion Expression and MDD Symptoms

\begin{tabular}{llll}
\hline Dependent measure: MDD symptoms (youth report) & & & \\
Variables included: & R Square Change & Beta & Significance \\
Inhibited (youth-report) & 0.246 & 0.429 & 0.000 \\
Disinhibited (youth-report) & 0.036 & 0.200 & 0.001 \\
Variables not included: & - & 0.009 & 0.879 \\
Inhibited (observed) & - & 0.057 & 0.308 \\
Disinhibited (observed) & R Square Change & Significance \\
Overall F (2, 227)=44.57, $p<001$, Adjusted R Square=0.276 & 0.000 \\
Dependent measure: MDD symptoms (parent report) & 0.052 & Beta & 0.229 \\
Variables included: & - & & 0.055 \\
Inhibited (youth-report) & - & 0.131 & 0.131 \\
Variables not included: & - & 0.097 & 0.077 \\
Disinhibited (youth-report) & 0.114 & \\
Inhibited (observed) & & \\
Disinhibited (observed) & & \\
Overall F (1, 228)=12.57, $p<001$, Adjusted R Square=0.048 & & \\
\hline
\end{tabular}


Table 3

Multivariate Tests of the Associations Between Emotion Expression and Impairment

\begin{tabular}{llll}
\hline Dependent measure: Impairment (interviewer report) & & & \\
Variables included: & R Square Change & Beta & Significance \\
Inhibited (youth-report) & 0.198 & -0.362 & 0.000 \\
Disinhibited (youth-report) & 0.055 & -0.249 & 0.000 \\
Variables not included: & - & -0.091 & 0.112 \\
Inhibited (observed) & - & -0.031 & 0.595 \\
Disinhibited (observed) & & & Significance \\
Overall F (2, 227)=38.41, $p<0.001$, Adjusted R Square=0.256 & 0.017 \\
Dependent measure: Impairment (parent report) & R Square Change & Beta & 0.023 \\
Variables included: & 0.027 & -0.156 & 0.221 \\
Inhibited (observed) & 0.022 & -0.148 & 0.146 \\
Inhibited (youth-report) & - & -0.081 & -0.100 \\
Variables not included: & - & & \\
Disinhibited (observed) & - & & \\
Disinhibited (youth-report) & & \\
Overall F (2, 227)=5.82, $p<0.01$, Adjusted R Square=0.040 & & \\
\hline
\end{tabular}

